\title{
Visual Discrimination, Categorical Identification, and Categorical Rating in Brief Displays of Curved Lines: Implications for Discrete Encoding Processes
}

\author{
David H. Foster \\ Department of Communication and Neuroscience \\ University of Keele, Keele, Staffordshire, England
}

\begin{abstract}
Visual discrimination, categorical identification, and categorical rating measurements were made on sets of curved-line stimuli drawn from a theoretically uniform continuum with curvature parameter $s$. In Experiment 1, discriminability of pairs of curved lines separated by a constant distance on the $s$ scale was measured at successive points along the scale. Curved lines were presented four at a time in a 100-msec display, which was followed by a random-dot mask. Discrimination performance was found to vary nonsmoothly with $s$. In Experiment 2, a categorical identification task was performed in which subjects labeled the curved-line stimuli of Experiment 1 straight, just curved, and more than just curved. From these data, a theoretical discrimination performance was computed that was closely congruent to the discrimination performance of Experiment 1. In Experiment 3, three different categorical rating scales with two, three, and four intervals were tested and each was shown to be less effective than the categorical identification scale for predicting discrimination performance. Mean ratings were, however, highly linear with $s$, suggesting that the curved-line continuum was psychometrically uniform. Experiment 4 provided further evidence for the uniformity of the curved-line continuum by measuring conventional acuity for curvature. Two rather than four curved lines were presented in each display; duration was increased to $2 \mathrm{sec}$; and the poststimulus mask was omitted. Acuity was found to vary linearly with $s$. It was concluded that under conditions in which attention is distributed over a number of elements in the field and in which viewing and effective visual processing time are restricted, performance in discriminating curved-line stimuli may be determined by relatively coarse, discrete visual processes.
\end{abstract}

Visual acuity for changes in the shape or arrangement of simple spatial stimuli is usually found to vary relatively smoothly and continuously over the range of stimulus variation. This applies, for example, to judgments of the spatial separation of two parallel lines as a function of their separation (Westheimer, 1979) and to other acuity tasks involving judg-

A portion of the data presented here was contained in a communication read at the London meeting of the Experimental Psychology Society, January 1982. I thank H. B. Barlow and P. M. A. Rabbitt for providing helpful comments on an initial report on Experiments 1 and 2, and S. R. Pratt and J. Thorson for critical review of the present article. I thank E. C. J. Eijkman for making available preprint material. I am grateful to $R$. Knapper for technical assistance.

Requests for reprints should be sent to David H. Foster, Department of Communication and Neuroscience, University of Keele, Keele, Staffordshire, ST5 5BG England. ments of collinearity and straightness of simple geometric figures as a function of their size (Andrews, Butcher, \& Buckley, 1973; Ludvigh, 1953; Westheimer \& McKee, 1977). The mechanisms limiting such performance are understood to be located at an early stage in the visual system, although they may be influenced by more central factors (for reviews, see Barlow, 1981; Westheimer, 1981).

Acuity performance of this kind is usually obtained with relatively long-duration displays (e.g., .2-2 sec) and with a small number of elements in the stimulus field (and it is impaired if other stimuli are presented close to the acuity target; see Westheimer \& Hauske, 1975). When the conditions of stimulus presentation are made more complex, howeverfor example, when there are several stimuli to be observed and when the presentation time is brief-form-discrimination performance 
may change from being smooth and finegrained to being coarse and effectively discontinuous along the range of stimulus variation. Such performance has been reported in the discrimination of simple three-dot figures when their deviation from collinearity is varied systematically (Foster, 1979, 1982b) and in the discrimination of chevron figures as the angle between the connected lines is varied (Foster, 1980b). For the three-dot figures, the discontinuities were interpreted as evidence for the operation of discrete visual encodings relating to the collinearity or noncollinearity of the dot figures, and, for the chevron figures, for the operation of discrete visual encodings relating to the acuteness or obtuseness of the angle between two lines.

\section{Categorical Discrimination}

The discontinuous discrimination performance just described is sometimes referred to as being categorical. The notion of categorical discrimination has received different interpretations, particularly in the perception of speech sounds (Healy \& Repp, 1982; Macmillan, Kaplan, \& Creelman, 1977; Repp, Healy, \& Crowder, 1979; Studdert-Kennedy, Liberman, Harris, \& Cooper, 1970). In the present work, this term is applied to performance in which, along some physically uniform stimulus continuum, the visual discriminability of pairs of equally spaced stimuli drawn from that continuum shows sharp maxima (and possible sharp minima). Sharpness may be quantified by determining extrema in the second-order difference quotient of the discrimination performance (see, e.g., Foster, 1982b) after normalizing the scale of the continuum with respect to the discrimination interval involved.

Data on categorical modes of discrimination have previously been reported in some highlevel form-perception tasks, for example, in learning to attach arbitrary speechlike sounds to various circular sectored figures (Cross, Lane, \& Sheppard, 1965), in discriminating closed-curve figures that admitted pictorial interpretations (Cermak, 1977; Shepard \& Cermak, 1973), and in learning geometric shapes (Rosch; 1973). Data on categorical modes of discrimination in low-level formperception tasks have, apart from the previ- ously cited studies, been obtained mainly in experiments concerned with grouping effects in the perception of displays of geometric elements forming textures (see, e.g., Beck, 1972; Fox, 1978; Julesz, 1971, 1980; Olson \& Attneave, 1970). Pomerantz (1978) has used displays with just four elements to investigate the relative primacy of lines and angles in form perception. Beck and Ambler (1973) have explored the effects of a number of elements to be attended to on peripheral sensitivity to line orientation and arrangement.

In auditory perception, the notion of categorical discrimination, sometimes epitomized as the "phoneme-boundary effect" (Wood, 1976), is well established (Liberman, Harris, Hoffman, \& Griffith, 1957; Liberman, Harris, Kinney, \& Lane, 1961; for reviews, see Macmillan et al., 1977; Massaro, 1976). There, the auditory discriminability of pairs of speechlike sounds from some appropriate continuum is found to be high when the sounds fall on opposite sides of a phoneme boundary and low when they fall within the same phoneme category. Categorical auditory discrimination has also been reported for nonspeechlike sounds (Cutting \& Rosner, 1974).

The status of categorical discrimination in auditory perception has been the subject of some discussion; most concern has centered on the question of the physical uniformity of the stimulus continuum and the contribution of continuous stimulus attributes to discrimination performance (Macmillan et al., 1977). As becomes apparent later, these considerations are also germane to the present work.

\section{Curved-Line Encodings}

This study is concerned with the possibility of discrete processes mediating the visual discrimination of curved lines when presented several at a time in a brief display. A priori, one might hope to detect discontinuities in visual discrimination corresponding perhaps to categories designating stimuli as straight and curved. In one study that included such a test, Beck (1973) found little evidence for that kind of categorical behavior. Watt and Andrews (1982) have found evidence for two curvature-sensitive processes when efficiency (calculated with respect to an ideal processor of curvature) was measured for short lines 
presented under conditions designed to test hyperacuity performance.

In the present study, a continuum of curvedline figures was generated by a procedure that secured a "transformational uniformity" of the stimulus continuum (Foster, 1980b). The general aim was to measure visual discriminability of pairs of curved lines at various points along the continuum and to relate any maxima and minima in that performance to the operation of possible discrete encodings. These putative encodings were identified in separate experiments in which the stimuli were assigned to various categories, according to an appropriate labeling scheme. Discrimination performance was then computed on the assumption that the specified categories corresponded to the discrete encodings determining the original discrimination performance.

To deduce from such experiments that there exist discrete encoding processes of the kind hypothesized involves three prerequisites: (a) the uniformity of the stimulus scale; (b) the robustness of the algorithm used to relate labeling performance to discrimination performance; and (c) the uniqueness of the putative categorical encodings. Much of the experimental material presented here was concerned with the resolution of these issues.

\section{Organization of This Study}

Experiment 1 measured visual discriminability of curved lines presented four at a time in a brief display. A poststimulus mask was introduced to further limit or at least attenuate subsequent processing of the fading visual image (Eriksen \& Spencer, 1969; Estes \& Taylor, 1964, 1966; Lupker \& Massaro, 1979; Morotomi, 1981). The use of a poststimulus mask was not, however, critical to obtaining the type of performance reported below (Foster, Note 1). Curved lines drawn from both the full range of curved-line stimuli and the first half of that range were used to examine range effects.

Experiment 2 measured labeling performance for the same set of stimuli presented under the same conditions as in Experiment 1. Curved lines indicated by a poststimulus cue were categorically identified as a function of their position along the stimulus continuum. The labels used were straight, just curved, and more than just curved. A theoretical discrimination performance predicted by discrete encoding processes corresponding to these categories was computed and compared with observed discrimination performance obtained in Experiment 1 for both the full-range and half-range stimulus sets.

Experiment 3 tested the sensitivity of the predicted discrimination performance derived in Experiment 2 to changes in the labeling regime. Three separate rating experiments were performed in which the curved lines of the previous two experiments were labeled using two-, three-, and four-interval uniform rating scales. Each of these rating performances was converted into a theoretical discrimination performance and compared with the observed discrimination behavior for both the full-range and half-range stimulus sets. These rating data were also used to evaluate the apparent uniformity of the curved-line continuum.

Experiment 4 made a different test of uniformity of the curved-line continuum. This was done by carrying out the discrimination task of Experiment 1 under conditions designed to elicit performance characteristic of conventional visual acuity. As a control on the stability of the processes underlying the performance obtained in Experiment 1, that experiment was performed again after Experiment 4.

Overall, it is argued that the present experiments show evidence of the operation of discrete processes encoding a range of curvedline stimuli that from theoretical, psychometric, and psychophysical considerations formed a uniformly spaced continuum.

\section{Experiment 1: Discrimination in Arrays of Curved Lines}

In this experiment, curvature discrimingtion performance was measured at steps alor $\mathrm{g}$ the continuum of curved lines illustrated in Figure 1 (section a). The continuum was produced by fixing the two terminal members of the range and generating intermediate members by the action of a local 1-parameter group of transformations (see Foster, 1979, 1980b), which, in this case, amounted simply to a procedure of horizontal interpolation. Thus any curved line in the range could be considered 
as being a horizontally magnified (or demagnified) version of any other curved line. Each curved line is specified by the parameter $s$, which measures the perpendicular distance between its chord and the midpoint of the curved line (see the inset to Figure 1). Apart from the end members of the range, no curved line was an arc of a circle.

The reason for generating patterns in this way has been explained in Foster (1979, 1980b). In brief, such a procedure establishes a transformational uniformity over the continuum in that the same spatial transformation relates any pair of curved lines separated by the same distance along the scale, independent of where they are located (Foster, 1980b). Farrell and Shepard (1981) effectively applied the same procedure to continuously vary the degree of symmetry of polygon figures used for apparent motion stimuli.
In the discrimination task, square arrays of four curved lines were presented as illustrated in Figure 1 (sections $b$ and $c$ ). Three of the curved lines in each display were identical, each with curvature parameter $s-d s$ (or $s+$ $d s$ ), and one of the curved lines was "odd" in that it had curvature parameter $s+d s$ (or, respectively, $s-d s$ ). The increment $d s$ was fixed and independent of $s ; 2 d s$ constituted the discrimination step. For brevity, the value of the curvature parameter $s$ about which the increments $d s$ were made is called the reference value. In Figure 1 (section b), $s=.95$-minarc visual angle, $d s=.47 \mathrm{~min}$ arc; in Figure 1 (section c), $s=3.31 \mathrm{~min}$ arc, $d s=.47 \mathrm{~min}$ arc. In all cases, the chord length was $.2^{\circ}$ (12 min arc). The task of the subject was to indicate the position of the odd curved line, the position varying randomly from trial to trial.

Discrimination performance was thus mea-

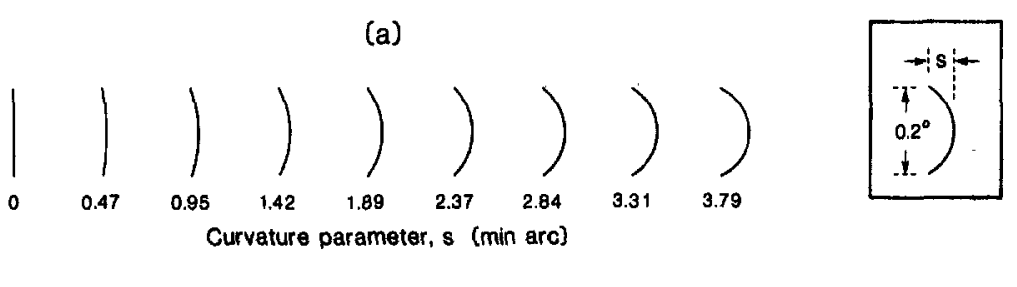

(b)

(c)

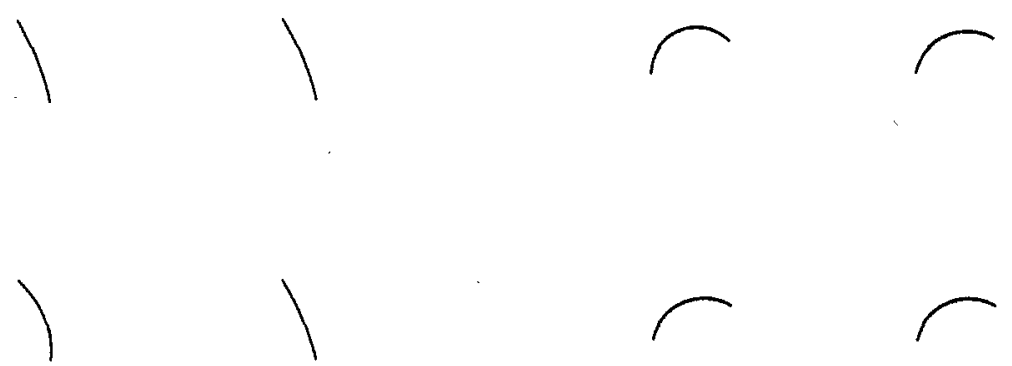

Figure 1. Examples of curved-line stimuli. (Section a shows a sequence of curved-line stimuli parameterized by curvature parameter $s$, which is measured in min-arc visual angle and defined as in the inset to the figure. The two terminal members of the family of curved lines [s $=0$ and 3.79 min arc] were arcs of circles, and intermediate members were generated by horizontal interpolation resulting from the action of a local 1-parameter group of transformations [see the Appendix]. Sections b and $c$ show typical stimulus displays [not to scale]. In section b the "odd" curved line had curvature parameter $s+d s=1.42 \mathrm{~min}$ arc, and the three identical curved lines had curvature parameter $s-d s=.47 \mathrm{~min}$ arc. In section $c$ the odd curved line had curvature parameter $s+d s=3.79 \mathrm{~min}$ arc, and the three identical curved lines had curvature parameter $s-d s=2.84 \mathrm{~min}$ arc. Each curved line had chord $12 \mathrm{~min}$ arc and line width approximately $1.2 \mathrm{~min}$ arc. The horizontal or vertical center-to-center separation of one curved line from another in each display was $2.0^{\circ}$.) 
sured with fixed increment $d s$ for reference values $s$ varying over the range .47 to 3.31 min arc. ${ }^{1}$ Performance was measured by the percentage correct, rather than reaction time (RT). The interdependence of the two measures is discussed in the Results section.

The number of curved lines in each display (four) was chosen on the basis of data derived in earlier experiments: Using many more than four curved lines made the task too similar to that involved in texture discrimination, and using much less than four curved lines reduced the task to a conventional acuity measurement. (The effects of curved-line number on eliciting the kind of performance reported here is described elsewhere; see Foster, Note 1). Paradigms other than the present single-interval, four-alternative, forced-choice paradigm have been used in this context, but results appear not to depend critically on the type of forcedchoice procedure or on configurational effects (Foster, 1979).

\section{Range Effects}

It was already anticipated that stimulus range effects would be important in determining performance (Parducci, 1974). Preliminary experiments with the full range of curved-line stimuli (Figure 1, section a) suggested that the selected sampling density (approximately .47-min-arc step size) of reference values of the curvature parameter $s$ in conjunction with range effects was not optimum for revealing possible discrete discrimination performance at low values of the curvature parameter $s$ (compare Braida \& Durlach, 1972). Additionally, in three-dot discrimination experiments (Foster, 1979, 1982b), discrimination performance was evident over deviations from collinearity corresponding to the first half of the present curvature range. To increase the density of reference values would have made experimental runs protracted. Instead, two experments were performed, the one with the full stimulus range as in Figure 1 , (section a), with reference values of $s$ at approximately .47-min-arc intervals, and the other with the first half of the stimulus range, with values of $s$ at approximately .22-min-arc intervals (starting at $.47 \mathrm{~min}$ arc). In both cases, the increment $d s$ was fixed at $.47 \mathrm{~min}$ arc. An advantage of this design for evaluating range effects in discrimination performance and, more important, for testing consistency in predicted discrimination performance becomes apparent later.

\section{Method}

Subjects. Four subjects, aged 26 to 36 years, participated in the experiment. Two of the subjects were female, R.S.S. and F.M.F., and 2 were male, R.K. and D.H.F. (the author). Each has normal or corrected-to-normal visual acuity (Snellen acuities each not worse than 6/5). R.S.S., F.M.F., and R.K. were unaware of the purpose of the experiment.

Stimuli and apparatus. The examples of stimulus arrays illustrated in Figure 1 (sections b and $c$ ) are not to scale: The chord of each curved line subtended $.2^{\circ}$ (12 min arc) at the eye and was approximately $.02^{\circ}(1.2 \mathrm{~min}$ arc) wide; the horizontal, or vertical, center-to-center separation of one curved line from another in the array was $2.0^{\circ}$. The duration of each display was $100 \mathrm{msec}$. In each display, three of the curved lines had curvature parameter $s-d s$ (or $s+d s$ ), and the fourth had parameter $s+d s$ (or, respectively, $s-d s$ ). The value of $d s$ was fixed at $.47 \mathrm{~min}$ arc. $\mathrm{A}$ fixation cross (not shown in Figure 1, sections b and c) was present at the center of the array. The stimuli were white and appeared superimposed on a uniform white $7^{\circ} \times 7^{\circ}$ background field, $50 \mathrm{~cd} \cdot \mathrm{m}^{-2}$.

The intensity of the curved lines was adjusted by each subject at the beginning of each experimental session to be 10-times the luminance increment threshold (typically, $50 \mu \mathrm{cd} \cdot \mathrm{sec})$. This setting was achieved by introducing a 1.0-log-unit neutral density filter over the stimulus lines and adjusting their intensity to increment threshold on the unattenuated background. The poststimulus mask used in this and most subsequent experiments in this study consisted of four $.4^{\circ} \times .4^{\circ}$ random-dot patterns, each covering the area of the field occupied by each of the four curved lines. Each random-dot pattern comprised 36 dots, each approximately 1.2 -min-arc diameter, $500 \mu \mathrm{cd}$.

The stimuli were produced on the screen of an X-Y display oscilloscope (Hewlett-Packard, Type 1300A) with P4 sulfide phosphor (decay time $60 \mu \mathrm{sec}$ ) controlled by a minicomputer (CAI Alpha LSI-2) with vector-graphics (Sigma Electronics Systems QVEC 2150) line generator. Each curved line was composed of 10 concatenated straight-line segments, and over the range of curvatures used here appeared smooth to the eye. The spatial fidelity of the display was such that deviations of the stimuli from specified shape did not exceed .09 -min-arc visual angle, $9 \%$ of the discrimination step $2 d s$. For subsequent control experiments in which performance approached hyperacuity levels (Westheimer, 1981), the fidelity of the system was increased by an auxiliary optical system. Each curved line on the screen of the CRT was refreshed at 20 -msec intervals; thus, each line was drawn identically six times to make

\footnotetext{
${ }^{1}$ The minimum value of $s$ was constrained by the requirement that $s-d s$ should not be negative for positive $s$ and $d s$. Although comparisons among curved lines with curvatures of different signs were possible, additional complications could be introduced relating to the discriminability of symmetric figures (see Foster, 1979).
} 
up the nominal $100-\mathrm{msec}$ stimulus duration. This fine temporal structure of the stimuli could not be detected visually. Display duration was too short to permit deliberate scanning of the stimuli. The display screen was viewed binocularly at a distance of $1.7 \mathrm{~m}$ through a view tunnel and optical system, which produced the uniform background field.

Procedure and experimental design. Subjects were informed that each trial involved the presentation of a display consisting of four curved lines whose curvatures were variable and such that in some cases the lines straight. Three of the lines were identical and the fourth was different in that it was curved more or less than the other three. Subjects were instructed to indicate after the end of the presentation the location of the "odd" curved line. Subjects were encouraged to respond as quickly as was consistent with accuracy.

Subjects fixated the central fixation target, and, when ready, initiated a trial by pressing the appropriate switch on a push-button box interfaced to the computer. After a 500-msec delay, the curved-line display appeared for 100 msec; after a 100-msec blank field, the random-dot masking field appeared for $500 \mathrm{msec}$. The fixation target then disappeared. Subjects maintained central fixation during the presentation period. When the subject had signaled his or her response on a push-button box, the fixation target reappeared after about 2 -sec delay, indicating that the next trial could be started.

Trials were performed in sequences of 28 . In each such run, each curved line had one of seven different reference values of the curvature parameter $s$ (for the full range, .47 to $3.31 \mathrm{~min}$ arc, and, for the first half range, .47 to 1.89 min arc, see Figure 1, section a), and four different orientations $\left(22.5^{\circ}, 67.5^{\circ}, 112.5^{\circ}, 157.5^{\circ}\right.$ to the vertical). Orientations different from the vertical and horizontal, and $45^{\circ}$ and $135^{\circ}$ obliques, were chosen to avoid confounding effects due to the general improvement in acuity associated with vertical and horizontal axes (Hakiel, 1978; Onley \& Volkmann, 1958; Rochlin, 1955; Weene \& Held, 1966). The increments $+d s$ and $-d s$ occurred equally often. Each subject carried out 2 blocks of 8 runs on separate days. Each block was preceded by a practice run. The sequence of presentations in each run was chosen pseudorandomly but balanced over runs to offset stimulus carry-over effects and response bias by subjects. There were two types of block, comprising exclusively runs with the full range of curved-line stimuli or the first half of that range. The two types of block were interleaved. Subjects were not given feedback on their performance.

The long-term repeatability of curved-line discrimination performance was evaluated by performing the whole experiment again 1 year later. Results are reported in the General Discussion section.

\section{Results}

Figure 2, sections $a$ and $b$, shows the results of the curved-line discrimination experiment. In each figure, the percentage of correct responses, corresponding to the correct discrimination of the odd curved line, curvature parameter $s+d s$ (or $s-d s$ ), from the three identical curved lines, curvature parameter $s-$ $d s$ (or, respectively, $s+d s$ ), is plotted against

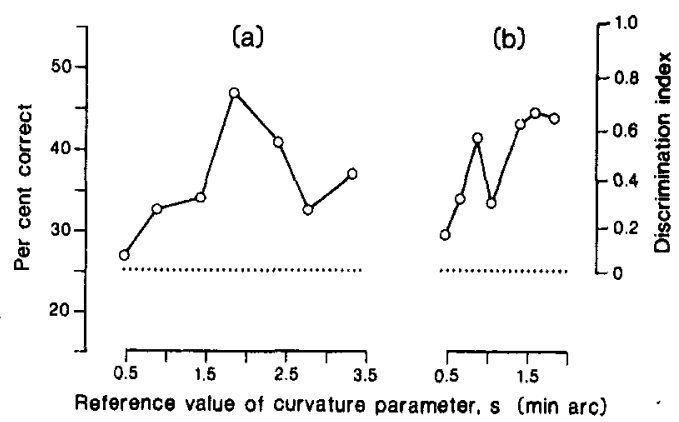

Figure 2. Performance in discriminating curved lines in an array of four lines. (The percentage of correct responses for discriminating the odd curved line, curvature parameter $s+d s$ [or $s-d s$ ], from the three identical curved lines, curvature parameter $s-d s$ [or, respectively, $s+d s$ ], is plotted against the reference value of the curvature parameter $s$ [see Figure 1]. The magnitude of the increment $d s$ was fixed at $.47 \mathrm{~min}$ arc. Data were pooled over four subjects. Performance in section a refers to the full range of curved-line stimuli [see Figure 1, section a], and in section $b$ to the first half of that range. Chance-level performance is shown by the horizontal dotted line.)

reference value of the curvature parameter $s$. Results are pooled over subjects, since individual performances were similar. The data in Figure 2 (section a) are for the full range of curved-line stimuli (see Figure 1, section a), and those in Figure 2 (section b) for the first half of that range. The magnitude of the increment $d s$ was fixed at $.47 \mathrm{~min}$ arc in all cases. Chance-level discrimination is shown by the horizontal dotted line. A scale transforming the percentage correct into discrimination index $d^{\prime}$ (Green \& Swets, 1966; Swets, 1973) is shown on the right ordinate.

For both the full-range and half-range stimulus sets, the dependence of discrimination performance on the reference value of the curvature parameter $s$ is manifestly nonconstant. For the full-range data (Figure 2, section a), there is a sharp maximum in the center of the scale, at $s=1.81 \mathrm{~min}$ arc (significant quadratic trend $^{2}, p<.05$ ); for the half-range data (Figure

\footnotetext{
${ }^{2}$ Because of truncation effects related to the resolution of the vector generator, the reference values of the curvature parameter $s$ were not precisely equally spaced. For the full-range stimulus set, maximum departure from ideal spacing was $4.9 \%$ of the discrimination step $2 d s$; for the half-range stimulus set (with twice the sampling density of the full-range set), maximum departure from ideal spacing was $9.4 \%$ of the discrimination step $2 d s$. These departures were sufficiently small to be neglected in the trend analysis. The assumption of precisely equal spacing was not required elsewhere in the analysis.
} 
2 , section $b$ ), there is a maximum at $.87 \mathrm{~min}$ arc, minimum at $1.03 \mathrm{~min}$ arc, and a subsequent rise (overall significant linear trend, $p<$ $.01)$. As anticipated, the half-range data show detailed structure not evident in the coarser sampling of the full-range data, although the gradient of the half-range data is not signifcantly different from the gradient of the first half of the full-range data $(p>.2)$.

Reaction time. Although RT was not used as the principal measure of performance, values were recorded to provide a general monitor of performance and to test for trade-off effects. For the full stimulus set, mean RT for a correct response was $631 \pm 33 \mathrm{msec}$; for the halfrange stimulus set, $560 \pm 25 \mathrm{msec}$ (here and subsequently: $M \pm 1 \mathrm{SEM}$ ). Mean RT for a correct response tended to decrease with increase in the percentage correct for the full stimulus set (gradient $-16.9 \pm 5.5 \mathrm{msec}$ percent $\left.^{-1} ; r=.81, p<.05\right)$. Thus, rather than there being a trade-off, the reverse occurred. No correlation was observed between mean RT for an incorrect response and the percentage incorrect $(r=.14, p>.2)$. For the half-range stimulus set, for which there was a much smaller range in RT values, no significant correlations emerged between mean RT and performance for either correct or incorrect responses $(r \leqslant .24, p>.2)$.

\section{Discussion}

Although the curved lines being discriminated in each display differed by a constant increment $2 d s$ in curvature parameter, discrimination performance varied strongly with $s$. When the results for the full-range and halfrange stimulus sets are taken together, two peaks are evidenced along the curvature continuum: the first at $s=.87 \mathrm{~min}$ arc, and the second at $s=1.81 \mathrm{~min}$ arc. These results suggest two questions. First, are they an artifact of some hidden nonuniformity in the curvature scale? Despite the theoretical rationale for scale uniformity, it might be argued that the peaks in discrimination performance shown in Figure 2 (sections $a$ and $b$ ) were the results of local expansions in the perceived curvature continuum, giving in turn an increase in the magnitude of the effective discrimination step $2 d s$. Second, if these peaks in discrimination performance were indeed the result of discrete encodings, then what are the characteristics of these encodings? Experiment 2 was directed to the second question. The third and fourth experiments were concerned with the question of scale uniformity.

\section{Experiment 2: Categorical Identification of Curved Lines}

Given that the curved-line discrimination performance shown in Figure 2 could be described in terms of discrete encoding processes, some possible candidates for these processes were tested in the present categorical identification ${ }^{3}$ experiment. Subjects assigned labels to the stimuli of Experiment 1 according to a fixed scheme. On the assumption that this labeling performance reflected the encodings underlying the discrimination performance of Experiment 1, a theoretical discrimination performance was calculated and tested against the observed performance.

The existence of two peaks, at $s=.87$ and $1.81 \mathrm{~min}$ arc along the curved-line continuum (Figure 2, sections a and b), suggested the existence of at least three discrete encodings (Foster, 1979). In view of the location of the peaks and the linear ordering of the stimuli along the continuum, the following three characterizations of these encodings seemed plausible: straight, just curved, and more than just curved. Accordingly, subjects were given these three categories to label the curved-line stimuli. To fully compare theoretical discrimination performance derived from the present categorization experiment with the observed discrimination performance of Experiment 1, labeling data were obtained with both the full range of curved lines (Figure 1, section a) and the first half of that range.

\section{Methods}

Subjects. The four subjects of Experiment 1 participated in this experment. R.S.S., F.M.F., and R.K. were unaware of the purpose of the experiment.

\footnotetext{
${ }^{3}$ The term categorical identification is used here to distinguish discrete labeling involving qualitative characteristics of the stimulus without reference to an equal-interval scale from discrete labeling involving quantitative characteristics defined with respect to some equal-interval scale. In so far as labeling under categorical identification was shown here to predict the distinguishability of the stimuli and presumably reflected their salient properties, the term is also appropriate in the sense of Durlach and Braida (1969).
} 
Stimuli and apparatus. The range of curved-line stimuli, their configuration; timing, and intensity were exactly as in Experiment 1. In each display three of the four curved lines were identical, each with curvature parameter $s-d s$ (or $s+d s)$, and one was odd, with curvature parameter $s+d s$ (or, respectively, $s-d s$ ). The poststimulus mask was also as in Experiment 1. In addition to the mask, however, a small arrow (of extent $.3^{\circ}$ ) appeared in the center of the display field, at the same time as the mask, pointing to the quadrant of the display previously occupied by the odd curved line. The odd curved line was thus cued $100 \mathrm{msec}$ after the display in which it occurred was extinguished (compare Lupker \& Massaro, 1979).

Procedure and experimental design. Subjects were shown an illustration of the full range of curved lines, as in Figure 1 (section a), or the first half of that range if that was to be used. Subjects were informed that each trial involved the presentation of a display consisting of an array of four curved lines of variable curvature and such that in some cases the lines were straight. Three of the curved lines in each display were identical, and the fourth was different in that it was curved more or less than the other three. After presentation of the curved-line display, a small arrow would appear in the center of the field at the same time as a random-dot masking field, the arrow pointing to one of the four curved-line locations (that of the odd curved line). Subjects were to report, in sequence, the appearance of the line pointed to and then that of any two other lines in the display, in terms of the following categories: (1) straight, (2) just curved, and (3) more than just curved. Responses were made on a push-button box interfaced to the computer. The three noncued curved lines were identical, and the choice of which two of these should be labeled was unimportant. In the discrimination task (see Experiment 1), the minimum number of curved lines required to make a correct estimate of the location of the odd curved line was three; hence the decision to label just that number here. Separate labeling experiments in which all four curved lines were identified gave similar results. Fixation conditions, initiation and time course of trials, and instructions concerning speed of responses were as in Experiment 1. The ordering and balancing of trials over runs were as in Experiment 1. The experiment was performed using the full range of curved-line stimuli and the first half of that range, also as in Experiment 1.

\section{Results}

Categorical identification. Figure 3 , sections $a$ and $b$, shows labeling performance for the three categories (1) straight, (2) just curved, and (3) more than just curved as a function of the curvature parameter $s$ of the curved line. The data in section a are for the full range of curved-line stimuli (see Figure 1, section a) and in section $b$ for the first half of that range. (The abscissa ranged from 0 to $3.79 \mathrm{~min}$ arc in section a and from 0 to $2.29 \mathrm{~min}$ arc in section $b$. The ranges are different from those in Figure 2, sections a and b, where the reference value of the curvature parameter $s$ was the independent variable.)

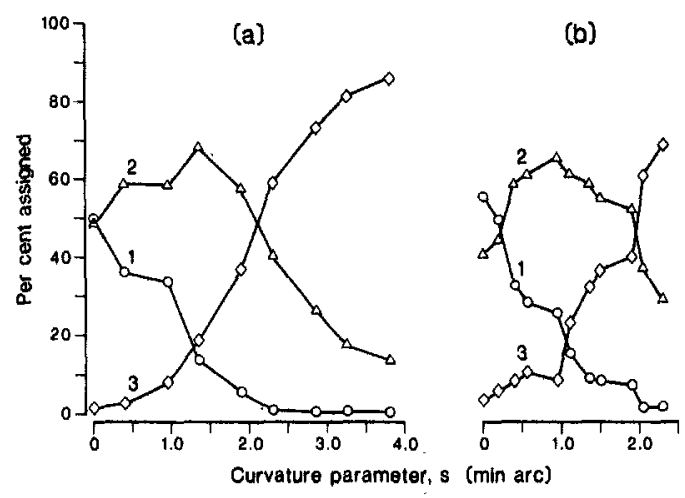

Figure 3. Performance in labeling curved-line stimuli according to a categorical identification scale with three categories (1) straight, (2) just curved, and (3) more than just curved. (The percentage of assignments to a given category is plotted against curvature parameter $s$. Data were pooled over four subjects. Performance in section a refers to the full range of curved-line stimuli and in section $b$ to the first half of that range.)

The percentage of responses assigning a given label to a given curved line is pooled over subjects whose performances were similar. The performances in Figure 3, sections a and b, although showing differences due presumably to range and density effects mentioned earlier, do exhibit regular behavior. Thus, the proportion of assignments straight decreases monotonically, and the proportion of assignments more than just curved increases monotonically with increasing curvature $s$ of the curved line (though for the half-range stimulus set, there is a small irregularity in the data at intermediate $s$ values); the middle category, just curved, is also used in a regular fashion in that the proportion of assignments first increases with increasing $s$ and then decreases. Notice that for zero-curvature lines $(s=0)$ the proportion of assignments straight and just curved was about the same (Figure 3, section a) and that for curved lines with $s$ values of up to $.56 \mathrm{~min}$ arc (Figure 3, sections a and b), reports of straight were assigned more than $30 \%$ of the time. This apparent nonveridicality of the stimuli was not novel. It is known that briefly presented simple geometric stimuli often appear distorted (Andrews et al., 1973). This occurrence is consistent with the notion that the stimuli gave rise to internal representations that were assigned on a probabilistic basis (Foster, 1979, 1980a, 1980b).

Repeatability. To test the repeatability of this labeling performance, 1 block of mea- 
surements ( 8 runs) was repeated by subjects R.S.S. and D.H.F. 4-6 weeks after the main experiment had been completed. After an allowance was made for possible en bloc displacements of assignment proportions along the $s$ scale of one sample step, that is, approximately $\pm d s$ for the full-range data or $\pm .5 d s$ for the half-range data, differences between repeated and original performance were not significant (for R.S.S., $\chi^{2}(2)=1.03, p>$ .5 ; for D.H.F., $\left.\chi^{2}(2)=.48, p>.5\right)$.

Predicted discrimination performance. In the Appendix, a summary is given of how predicted discrimination performance for the curved-line stimuli was computed from the categorization data. The principle underlying the computation is simple: The more likely that different internal encodings were given to different curved lines, the more likely that the lines would be discriminated (see Foster, 1982a, for a more detailed discussion). Detailed computations required a choice of metric to evaluate the differences in probabilities, but the form of the dependence of predicted discrimination performance on curvature parameter $s$ did not depend critically on that choice (see the Appendix and Figure Al, sections $a$ and $b$ ).

The hypothesis tested here is that the variation in assignment proportions with curvature parameter $s$ shown in Figure 3, sections a and $b$, reflects the actual assignment probabilities involved in determining performance in the discrimination task of Experiment 1. As explained in the Appendix, predicted discrimination performance is given by the sum over all categories of the absolute differences between assignment proportions obtained with each successive pair of curved lines. Under certain conditions, this sum may be reduced (Foster, 1980b) to give a simple correspondence between categorization and discrimination performances. This may be summarized as follows: Where assignment proportions are changing most rapidly, predicted discrimination performance is maximum, and where assignment proportions are maximum, predicted discrimination performance is minimum.

Figure 4 , sections $a$ and $b$, shows for the full-range and half-range stimulus sets predicted discrimination performance (broken lines) and actual discrimination performance from Experiment 1 (continuous lines). (The

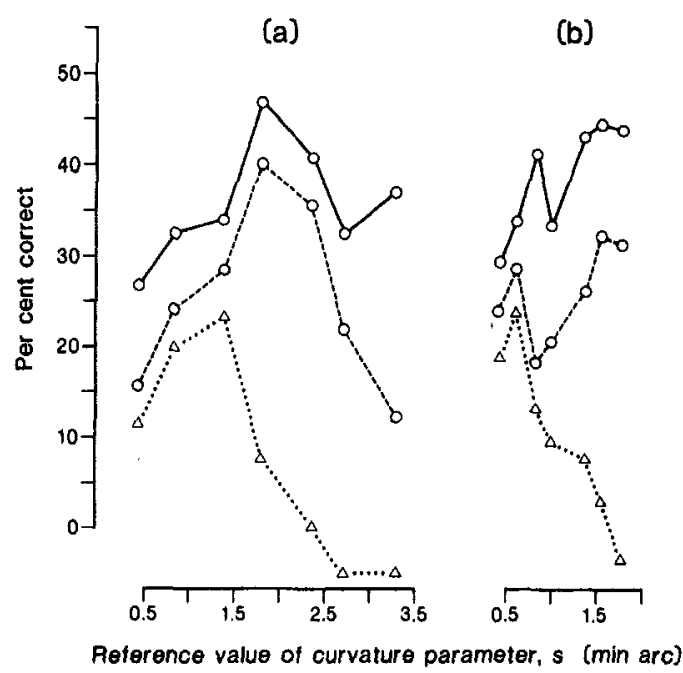

Figure 4. Predicted performance (open circles joined by broken lines) and observed performance (open circles joined by continuous lines, replotted from Figure 2) in discriminating curved lines. (The percentage of correct responses for discriminating the odd curved line, curvature parameter $s+d s$ [or $s-d s$ ], from the three identical curved lines, curvature paramter $s-d s$ [or, respectively, $s+d s]$ is plotted against the reference value of the curvature parameter $s$. Performance in section a refers to the full range of curved-line stimuli and in section $b$ to the first half of that range. Data for predicted discrimination performances were derived from the corresponding sets of categorical identification data of Figure 3 by means of the procedure described in the Appendix. Performances shown by the dotted line joining open-triangle symbols are predicted discrimination performances derived from the corresponding sets of categorical identification data of Figure 3 after merging the responses for the categories just curved and more than just curved. Predicted discrimination performances have been displaced vertically for ease of comparison. Data were pooled over the same four subjects for predicted and observed performances.)

dotted lines joining open-triangle symbols refer to a subsequent computation.) Predicted discrimination performances have been displaced vertically for ease of comparison.

The agreement between predicted and observed performance is good. For the full range of curved-line stimuli, predicted performance shows a central maximum, and, for the half range of curved-line stimuli, predicted performance shows a maximum at low $s$ values, a subsequent minimum, and an almost monotonic íncrease as $s$ increases. Most important, the effects of stimulus range on predicted discrimination performance parallel the effects of stimulus range on observed discrimination performance. 
In making more detailed comparisons of theoretical and observed discrimination performance, the following points should be noted.

1. The computational procedure used in the Appendix to obtain theoretical discrimination performance cannot set a level to the absolute value of expected discrimination performance. More generally, the computational procedure predicts performance only up to a monotonic scaling function (Foster, 1980b).

2. Although the positions of the minima in theoretical and observed discrimination performances shown in Figure 4 (section b) differ by $.173 \mathrm{~min}$ arc along the curvature parameter scale, this misalignment is just $17 \%$ of the discrimination step $2 d s$.

To quantify the match of theoretical and observed discrimination performances, a measure was used that takes into account some of the qualitative properties (e.g., related to maxima and minima) of the discrimination performances. Second-order difference quotients of the performances shown in Figure 4, sections $a$ and $b$, were computed and the resulting sets of data compared by a Pearson product-moment correlation coefficient, allowing for possible displacements of performance along the $s$ scale of one sample step (i.e., approximately $\pm d s$ in Figure 4 , section $a$, and approximately $\pm .5 d s$ in Figure 4 , section b). This procedure was designed to be sensitive to the presence of maxima and minima and insensitive to the relative scaling of performance level and to small "jitter" along the internal $s$ scale (see preceding test of repeatability). For the full-range data (see Figure 4 , section a), this correlation coefficient had a value of .96, and, for the half-range data, a value of .99 ( $p<.01$ for both). To show that this correlation measure also revealed poor matches of theoretical and observed discrimination performances, the theoretical performances of Figure 4 (sections a and b; broken lines) were interchanged and the fits to the observed performances recalculated. Correlation coefficients were .74 and .28 , respectively, clearly smaller than the original values of .96 and .99 , respectively.

\section{Discussion}

Performance in assigning the labels straight, just curved, and more than just curved to the curved-line stimuli of Experiment 2 provided a satisfactory basis for estimating assignment probabilities of discrete encodings supposed to underlie the discrimination performance of Experiment 1. The agreement between predicted and observed discrimination performance was evident both in the characteristics of the performances and in the effects of stimulus range.

It should perhaps be stressed that although the present categorical identification experiment and the previous discrimination experiment had the same stimulus ranges, display configuration, and timing, there are two important features that distinguish the two experiments. First, in the discrimination experiment, no explicit identification of the curvedline stimuli was required, only the location of the "odd" curved-line stimulus. In contrast, in the categorical identification experiment, the odd curved line did not have to be located; rather, its position was cued and subjects had to report on its appearance (and that of other lines in the array). Second, the categorical identification data could not yield discrimination performance estimates unless specific computational assumptions were made concerning the hypothesized processes governing the encoding of curved-line stimuli (see the Appendix):

As to the choice of categorical labels, straight, just curved, and more than just curved, subjects found the labeling task easy and assigned stimuli to the prescribed categories in a regular fashion. It might be argued that the choice of labels in the categorical identification task was based on ad hoc phenomenological considerations. The justification for using these "natural" categories depends, however, on their adequacy in generating accurate predictions of discrimination performance. The same test of appropriateness could, of course, be applied to any attribute of the curved-line stimuli that might be used by the visual system in establishing an internal representation of the curved lines in the discrimination experiment. For example, just two categories, say straight and curved, might be thought to suffice. One informal indication of the adequacy of two such categories can be obtained from the data of Figure 3 (sections $a$ and $b$ ) by merging the responses for categories just curved and more than just curved. In Figure 4 (sections a and b), the dotted lines joining open-triangle sym- 
bols show the predicted discrimination performance. The fit is manifestly poorer than that obtained with the unmerged categorizations (correlation coefficients, computed as before, were .43, $p>.2$, and .93, $p>.05$, for the full-range and half-range data, to be compared with the original values of $.96, p<.01$, and $.99, p<.01$, respectively). This merging procedure need not of course yield the same data as that obtainable experimentally with a two-category scale. This leads to a more general question: Would the experimental use of other types of categorical scale give acceptable predictions? To test this notion, the following labeling experiment was performed using three different categorical rating scales.

\section{Experiment 3: Categorical Ratings of Curved Lines}

A categorical rating scale with equal intervals provides an obvious alternative to the previous, unequally spaced, categorical identification scale for predicting discrimination performance. Rationales for deciding on a particular number of steps or intervals in a scale have varied (see, e.g.; Guilford, 1954, p. 289); in the present study, the natural course was to vary the number of intervals to bracket those of the previous experiment. Thus, this experiment was similar to the previous one except that instead of labeling the curved-line stimuli in terms of the categories straight, just curved, and more than just curved, subjects performed a standard rating task using two-, three-, and four-interval scales. This set of scales was adequate for testing interval-number effects. It should be noted, however, that a categorical rating scale with many more than four intervals or a continuous rating or magnitude-estimation scale would be unlikely to provide an adequate basis for predicting discrimination performance by the method outlined in the Appendix. This is because for each value along the rating scale, the proportion of times it is assigned must be defined for each $s$ value. For scales with large numbers of intervals or values, there would be many poorly sampled rating values.

The principal aim, then, of the experiment was to determine which, if any, of these three equal-interval rating scales would provide predicted discrimination performance comparable to that given by the categorical identification scheme of Experiment 2.

\section{Method}

Subjects. The four subjects of Experiments 1 and 2 participated in this experiment. R.S.S., F.M.F., and R.K. were unaware of the purpose of the experiment.

Stimuli and apparatus. The stimuli and apparatus were identical with those of Experiment 2.

Procedure and experimental design. Subjects were shown an illustration of the full range of curved lines, as in Figure 1 (section a), or the first half of that range if that was to be used. Subjects were informed that each trial involved the presentation of a display consisting of an array of four curved lines of variable curvature and such that in some cases the lines were straight. Three of the curved lines in each display were identical and the fourth was different in that it was curved more or less than the others. After presentation of the curved-line display, a small arrow would appear in the center of the field at the same time as the random-dot masking field, the arrow pointing to one of the four curved-line locations (that of the odd curved line). Subjects were to report, in sequence, the appearance of the line pointed to and then that of any two other lines in the display using, for the four-interval scale, the labels $1,2,3,4$, where 1 represented lines of least curvature and 4 represented lines of most curvature. These instructions were modified correspondingly when the three-interval and the two-interval rating scales were used. It was emphasized to the subject that the classification should be such that the categories were of equal width on his or her curvature scale, even though the total number of distinguishable curved lines in the range presented might exceed the number of response categories given.

Responses were made on a push-button box interfaced to the computer. Fixation conditions, initiation and time course of trials, and instructions concerning speed of responses were all as in Experiment 2, as were the ordering and balancing of trials over runs. These three rating experiments were each performed with the full range of curved-line stimuli and with the first half of that range, in separate blocks, the ordering of which was counterbalanced over subjects. The ordering of blocks according to number of rating intervals was also balanced over subjects.

\section{Results}

Categorical rating. Sections $\mathrm{a}, \mathrm{c}$, and $\mathrm{e}$ in Figure 5 show the results of the three sets of categorical ratings for the full range of curvedline stimuli; sections $b, d$, and $f$ in Figure 5 show the corresponding results for the first half of that range. The percentage of responses assigning a given rating from the two-, three-, and four-interval scales, pooled over subjects, is shown as a function of the curvature parameter $s$.

The rating data for the full range of curvedline stimuli show the expected distributions with all three rating scales. For the two-interval scale (Figure 5, section a), the boundaries of the rating intervals (defined by the points at which rating proportions for separate cate- 
gories cross over) are at about $2.1 \mathrm{~min}$ arc, approximately half way along the full range; for the three-interval scale (Figure 5, section c), the distributions of responses are also approximately even along the curvature scale,

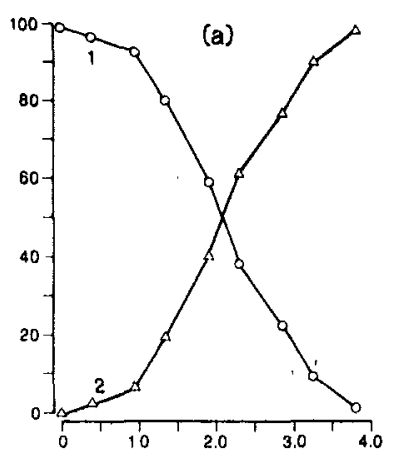

(b)
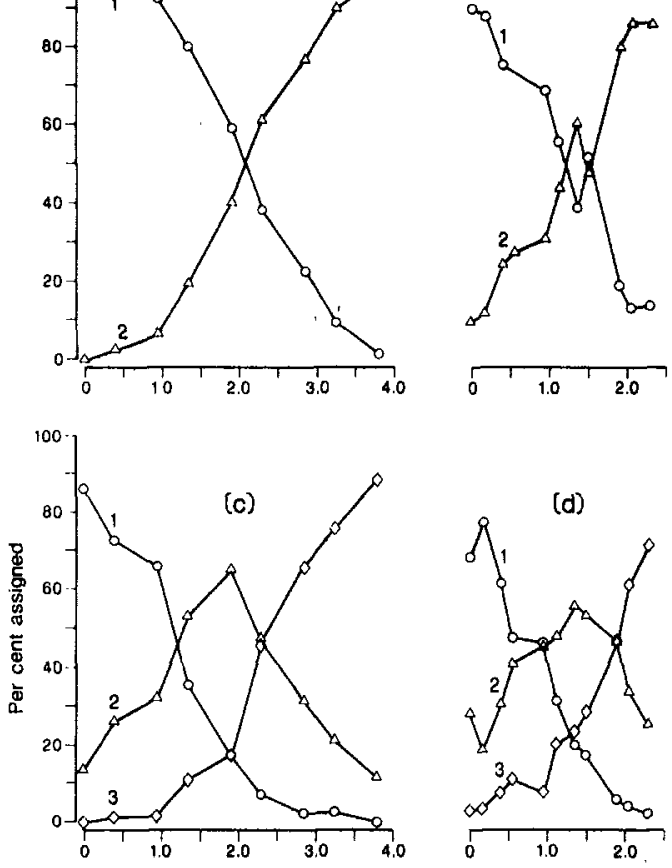

(d)
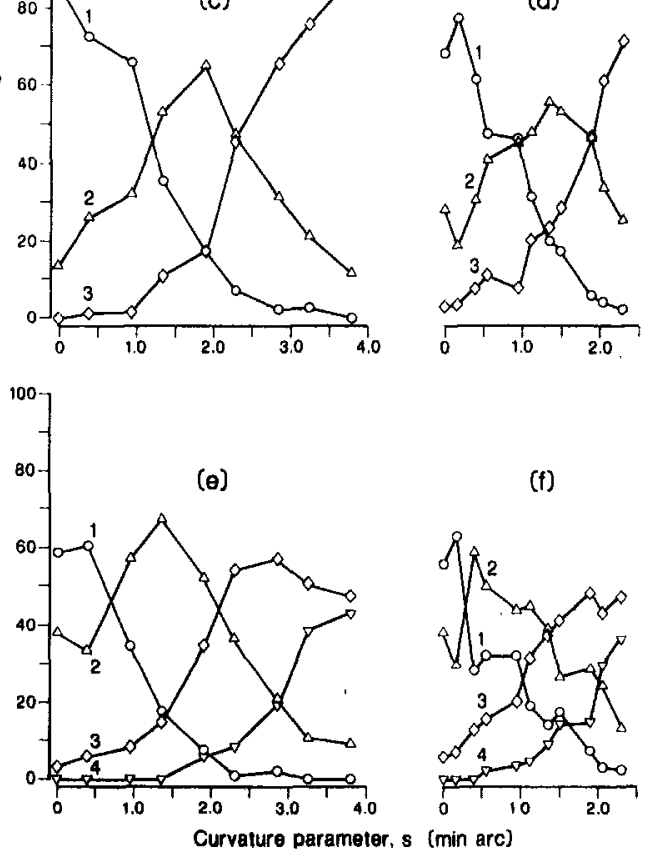

Figure 5. Performance in labeling curved-line stimuli according to three equal-interval, categorical rating scales: in sections $\mathrm{a}$ and $\mathrm{b}$ for $\mathrm{a}$ two-interval scale (labels 1 and 2), in sections $\mathrm{c}$ and $\mathrm{d}$ for a three-interval scale (labels 1 , 2,3 ), and in sections $e$ and $\mathbf{f}$ for a four-interval scale (labels $1,2,3$, and 4). (The percentage of assignments to a given category is plotted against curvature parameter $s$. Data were pooled over four subjects. Performances in sections $a, c$, and e refer to the full range of curved-line stimuli, and in sections $b, d$, and $f$ to the first half of that range.) with boundaries at about $1.2 \mathrm{~min}$ arc (rating 1 to 2 ) and $2.3 \mathrm{~min}$ arc (rating 2 to 3 ); for the four-interval scale (Figure 5 , section e), the distributions are a little less even, the boundaries being at about $.7 \mathrm{~min}$ arc (rating 1 to 2), $2.1 \mathrm{~min}$ arc (rating 2 to 3 ), and $2.9 \mathrm{~min}$ arc (rating 2 to 4 ).

The rating data for the half range of curvedline stimuli (Figure 5, sections $b$, d, and $f$ ) show similar, though not identical, dependencies on $s$. The variation of percentage of assignments of a given rating vary less smoothly with curvature parameter $s$ than for the full range of curved-line stimuli. This result is not unexpected because the curved lines being rated are sampled at steps along the curvedline continuum that are about one quarter of the discrimination step $2 d s$.

It is instructive to compare the rating data for the three-interval scale (Figure 5, sections $c$ and d) with the categorical identification data (Figure 3, sections a and b, respectively) from Experiment 2. The number of categories in each case was the same, and the two experiments differed only in the way that the categories were defined. For both full-range and half-range data, the redistribution of responses between categories 1 and 2 is evident at low $s$ values. Under the nonuniform categorical identification instructions, the categories 1 (straight) and 2 (just curved) were treated almost indistinguishably for $s$ values up to .5 min arc (Figure 3). In contrast, under the uniform rating-scale instructions, the categories 1 and 2 were treated very differently over that range (Figure 5, sections $\mathrm{c}$ and d). Overall, for the full-range data, proportions of responses assigned to categories 1 and 2 were $13.4 \%$ and $44.1 \%$, respectively, with the categorical identification scale, and $28.3 \%$ and $36.2 \%$, respectively, with the rating scale. For the half-range data, there was a similar pattern. The proportions of assignments to categories 1 and 2 were $19.4 \%$ and $52.5 \%$, respectively, with the categorical identification scale and $32.8 \%$ and $41.4 \%$, respectively, with the rating scale. The reason for the redistribution of responses is apparent. In the categorical identification experiment, the categories 1 and 2 corresponded to the designations straight and just curved, which would have forced assignments to category 1 to cover predominantly a small section at the beginning of the curvature range (see 


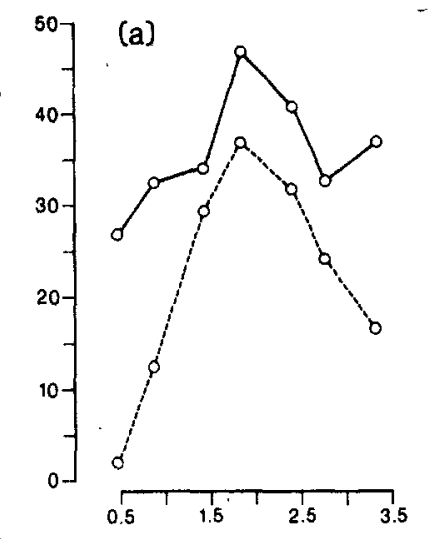

(b)
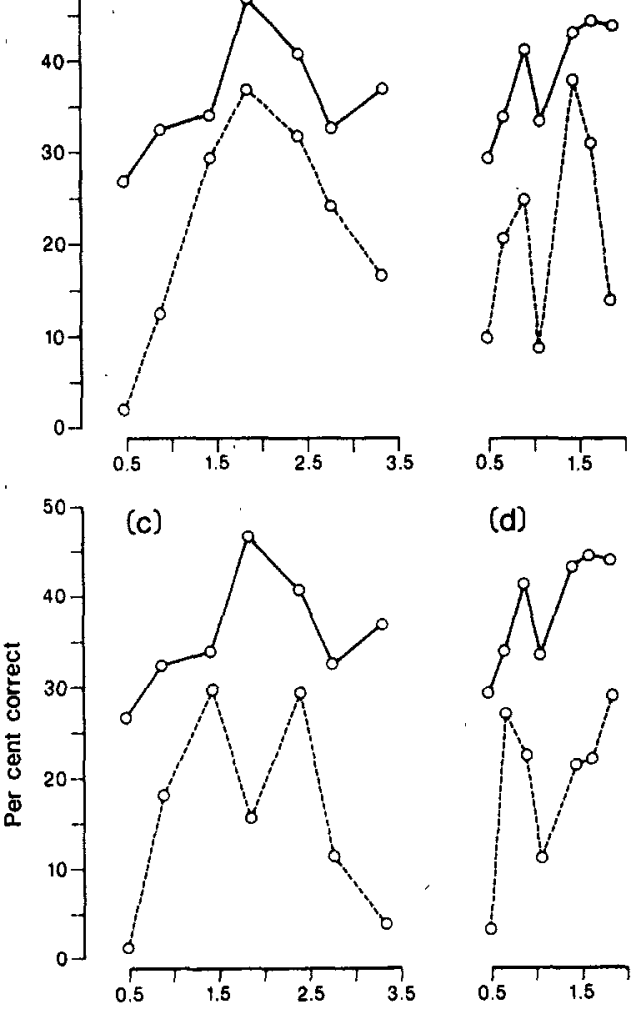

(d)
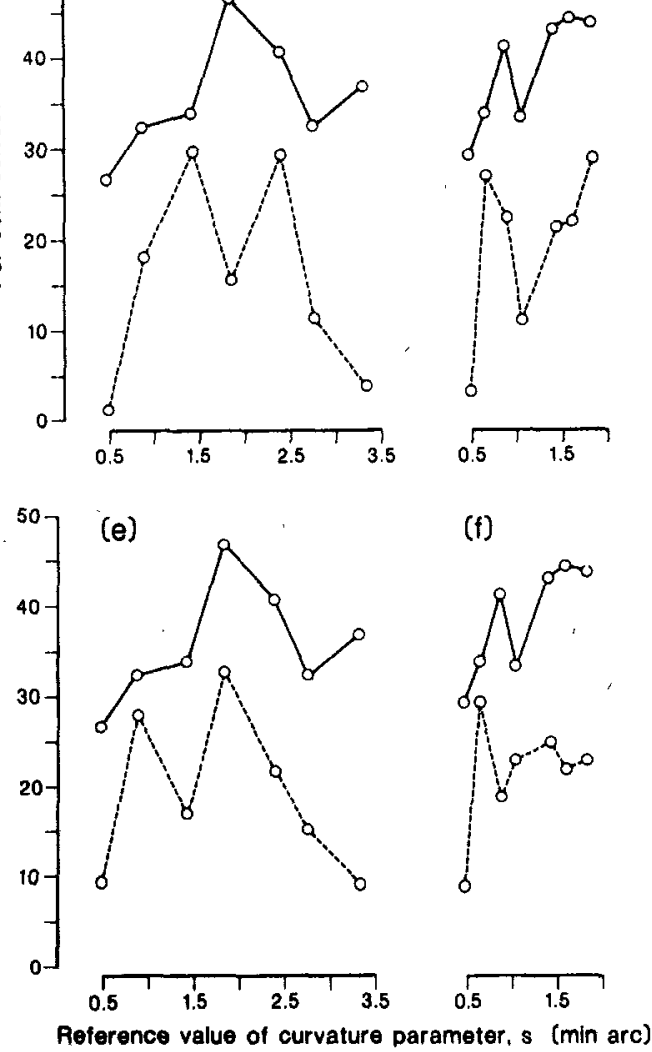

Figure 6. Predicted performance (broken line) and observed performance (continuous line, replotted from Figure 2) in discriminating curved lines. (The percentage of correct responses for discriminating the odd curved line, curvature parameter $s+d s$ [or $s-d s$ ], from the three identical curved lines, curvature parameter $s-d s$ [or, respectively, $s+d s]$ is plotted against the reference value of the curvature parameter s. Data for predicted discrimination performances were derived from the corresponding sets of categorical rating-scale data of Figure 5 by means of the procedure described in the Appendix. Performances in sections $a, c$, and $e$ refer to the full range of curved-line
Experiment 4), and, by the nature of the thresholds involved, an overlap with assignments to category 2 at low $s$ values. With the uniform rating scale, categories 1 and 2 (and 3 ) were required to be of equal size, which would have given a proportion of assignments to category 1 greater than that obtained with the categorical identification scale, and little overlap between assignments to categories 1 and 2 at low $s$ values. The extent of the uniformity of all three rating scales is dealt with later.

Predicted discrimination performance. By means of the computational procedure summarized in the Appendix, theoretical discrimination performances based on these sets of categorical rating data were computed exactly as in the preceding Experiment 2. In Figure 6 , sections a, c, and e and sections $b, d$, and f show, for the full-range and half-range stimulus sets, respectively, predicted discrimination performances (broken lines) for the three rating scales. For comparison, observed discrimination performances obtained in Experiment 1 are also shown (continuous lines). Predicted discrimination performances have been vertically displaced for ease of comparison.

It is obvious that when the results for the full-range and half-range stimulus sets are taken together, the two-, three-, and four-interval rating scales each gave rise to a predicted discrimination performance that fitted the observed performance less well than that obtained from the categorical identification data of Experiment 2. For example, the double peaks derived for the three- and four-interval rating scales for the full curvature ranges (Figure 6 , sections $c$ and e) are clearly incompatible with the observed performance.

These informal comparisons are supported by a correlation analysis similar to that used

stimuli, and those in sections $b, d$, and $f$ to the first half of that range. The predicted performances in sections a and $b$ were obtained from the two-interval scales [Figure 5 , sections $a$ and $b]$, the predicted performances in sections $\mathrm{c}$ and $\mathrm{d}$ from the three-interval scales [Figure 5, sections $c$ and d], and the predicted performances in sections $e$ and $f$ from the four-interval scales [Figure 5 , sections $e$ and $\mathrm{f}]$. Predicted discrimination performances have been displaced vertically for ease of comparison. Data were pooled over the same four subjects for predicted and observed performances.) 
Table 1

Comparison of Theoretical and Observed Discrimination Performances

\begin{tabular}{lcl}
\hline \multicolumn{1}{c}{ Scheme } & Full scale & Half scale \\
\hline Two-interval rating & .70 & $.88^{*}$ \\
Three-interval rating & .84 & .51 \\
Four-interval rating & .80 & $.99^{* *}$ \\
$\begin{array}{l}\text { Three-category } \\
\text { identification }\end{array}$ & $.96^{* *}$ & $.99^{* *}$ \\
\hline
\end{tabular}

Note. Maximum correlation coefficients are given for second-order difference quotients of theoretical and observed discrimination performances after relative shifts along the curvature scale of not more than one sample interval.

$* p<.05$. * $p<.01$.

in Experiment 2. Second-order difference quotients of each of the hypothetical discrimination performances shown in Figure 6 were computed and compared with those for the observed discrimination performances. Table 1 summarizes these results and also includes the results for the previous categorical identification experiment. This evaluation confirms the results of informal inspection. Discrimination performance predicted from the categorical identification data provided a better fit to observed performance than did any of the predicted performances from the ratingscale data. When the assignments to the two highest categories of the four-interval rating scale were merged to form a nonuniform threeinterval scale, rather like the categorical identification scale of Experiment 2, the resulting fit of predicted discrimination performance to observed performance was little changed (correlation coefficients of .81 and .99 for the fullrange and half-range data, to be compared with the original values of .80 and .99 , respectively).

Rating-scale uniformity. How uniform were the two-, three-, and four-interval rating scales? To answer this the mean category rating was computed as a function of the curvature parameter $s$ for each of the three rating scales.

In Figure 7, sections a, c, and e and sections $b, d$, and $f$ show for the full-range and halfrange sets of curved-line stimuli, respectively, mean category rating plotted against $s$ for each of the two-, three-, and four-interval rating scales. In each graph, the ordinate shows the full range of category ratings available. The straight lines in each graph are least squares regression fits. The proportion $w^{2}$ of variance accounted for by each fit is indicated.

There was slightly more scatter of mean ratings about the regression lines for the halfrange data, which may be a consequence of the smaller sampling intervals on the $s$ scale. The greater variability of the data for the twointerval scale is presumably because of the coarseness of that scale. Nevertheless, in all cases, the proportion of variance accounted for does not fall below $95.1 \%$, and the linearity of the four-interval scale is extremely high $\left(w^{2}=98.1 \%\right)$.
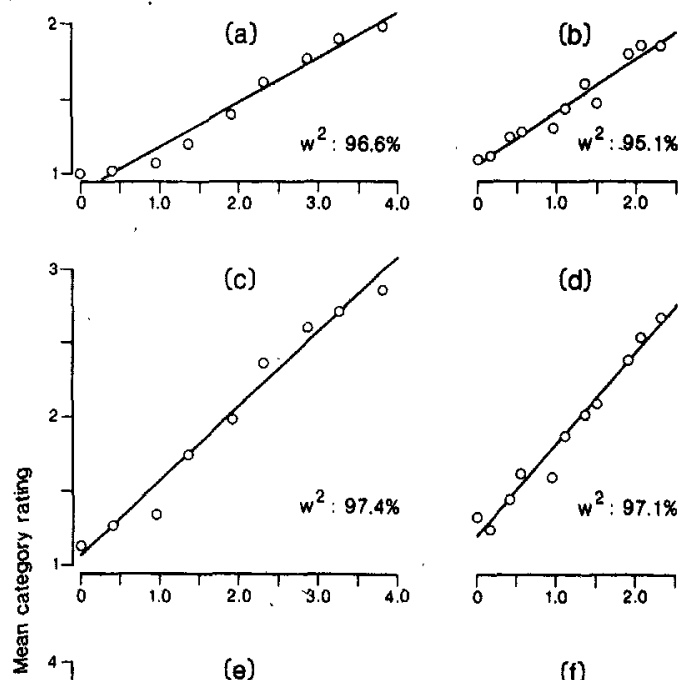

(d)

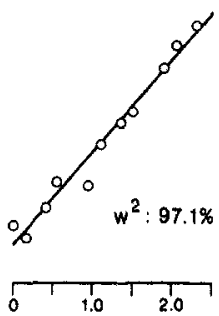

(f)

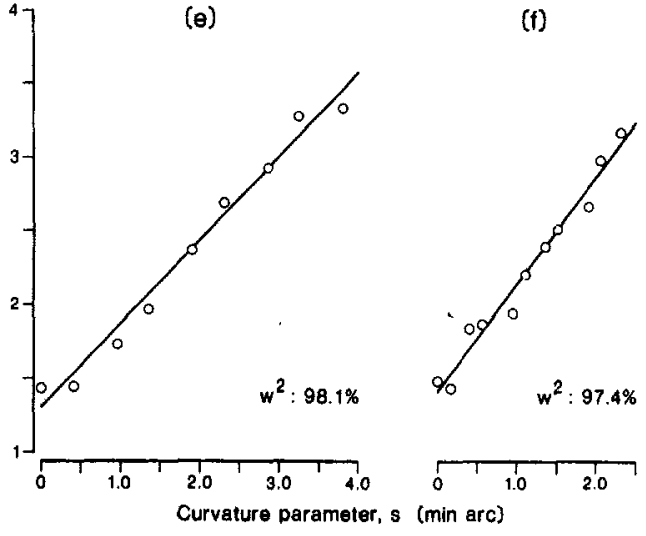

Figure 7. Rating-scale uniformity for the three categorical rating scales: in sections $a$ and $b$ for the two-interval scale, in sections $\mathrm{c}$ and $\mathrm{d}$ for the three-interval scale, and in sections $e$ and $f$ for the four-interval scale. (Mean category rating is plotted against curvature parameter s. Proportion $w^{2}$ of variance accounted for by a linear fit is indicated. Performances in sections a, $c$, and e refer to the full range of curved-line stimuli, and those in sections $b, d$, and $f$ to the first half of that range.) 
As a control for the appropriateness of this linear model for the mean rating data, regression fits were calculated for a number of other plausible models (see, e.g., Poulton, 1979; Stevens, 1974) with the aid of a computerbased modeling technique (GLIM; Baker \& Nelder, 1978). These models included logarithmic transformations of the curvature parameter $s$ and of mean rating values, and polynomial functions of the third degree in $s$. For those models that had the same degrees of freedom as the original linear model, fit was either worsened or not improved noticeably.

\section{Discussion}

This experiment has two principal implications. First, data from each of the three rating scales provided a rather poorer basis for predicting discrimination performance than did the original categorical identification data. This mismatch between the rating scale performance and the original discrimination performance was unlikely to be the result of a general drift in the characteristics of otherwise identical processes underlying the two sets of data. As reported in the General Discussion section, discrimination performance measured again after the following experiment appeared unchanged. Second, subjects used these rating scales as instructed, in that they distributed their responses so that all scales (two-, three-, and four-interval) had approximately equal apparent intervals. The fact that mean ratings varied linearly with the curvature parameter is particularly relevant because it lends support to the thesis that the nonsmooth discrimination performance obtained in Experiment 1 was not an artifact of some nonuniformity in the curved-line continuum.

Because the question of the linearity or, less restrictively, the smoothness of the curved-line continuum was critical to the demonstration of categorical discrimination, one further test of scale smoothness was made in the following experiment.

\section{Experiment 4: Acuity for Curvature}

The conditions of stimulus presentation were chosen in Experiment 1 to favor the use of discrete encoding processes. Thus stimulus displays contained several (four) curved lines to be attended to, were presented for short durations ( $100 \mathrm{msec})$, and were followed by a poststimulus mask to disrupt scrutiny of the fading visual image. If the observed peaks and troughs in discrimination performance (see Figure 2) were indeed an artifact of the nonuniformity of the curved-line continuum not revealed in the previous rating experiments, then such irregularities should also be manifested when discrimination performance was measured under conditions eliciting conventional acuity performance. In this experiment, the number of curved lines in each display was reduced to two, the duration of each display was increased to $2 \mathrm{sec}$, and there was no poststimulus mask. Under these conditions, factors related to visual processing time and distribution of attention should not set limits on performance (see, e.g., Beck \& Ambler, 1972, 1973).

Note that the intention here was merely to contrast discrimination performance that was obtained under one set of conditions known to facilitate a discrete mode of operation with discrimination performance that was obtained under a different set of conditions known to facilitate a continuous mode of operation. The relative contributions of factors such as numbers of figures in the display, display duration, and mask delay in determining, for example, peripheral discrimination performance have been investigated in detail by Beck and Ambler $(1972,1973)$. The particular role of these factors in determining performance of the kind described here is to be the subject of a separate study.

\section{Method}

Subjects. The same four subjects of the previous experiments participated in this experiment. R.S.S., F.M.F., and R.K. were unaware of the purpose of the experiment.

Stimuli and apparatus. The stimuli and apparatus were similar to those of Experiment 1, except for the following modifications. Two, rather than four, curved lines, the one with curvature parameter $s+d s$ and the other with $s-$ $d s$, were presented in each display and positioned $1.4^{\circ}$ horizontally to the left and to the right of the fixation target. Their eccentricity was the same as that of the curved lines in Experiment 1. The duration of the display was 2 $\mathrm{sec}$, and there was no poststimulus mask. The intensity of the curved-line stimuli was adjusted as in Experiment 1 , and other conditions of viewing were also the same.

From exploratory measurements, it was known that the threshold for detecting changes in curvature was about three times lower under these conditions than under the conditions of Experiment 1 . To achieve the appropriate spatial fidelity in the displayed curves, an auxiliary optical 
system was introduced to locate the stimuli in the visual field so that maximum precision could be given by the vector generator to curve construction. Departures of the stimuli from specified shape did not exceed $.02 \mathrm{~min}$ arc, $7 \%$ of a discrimination step $2 d s$.

Procedure and experimental design. Subjects were informed that each trial involved the presentation of a display consisting of two curved lines of variable curvature, one line curved more than the other. They were to indicate at the end of the presentation the location (left or right) of the more curved line. (With only two curved lines in the display, an "odd" curved line could not be defined as in Experiment 1, but, because there were two curved lines in each display, the task was essentially symmetric with respect to sign of curvature.) Throughout the presentation period, subjects fixated the central fixation target. Initiation and recording of responses were as in Experiment 1. Subjects were encouraged to respond as quickly as was consistent with accuracy. The range of reference values of the curvature parameter $s$ and the orientations of the curved lines were also as in Experiment 1. The magnitude of the increment $d s$ in $s$ was fixed at $.17 \mathrm{~min}$ arc $(10.1 \mathrm{sec}$ arc), approximately one third of the value used in Experiment 1. As in all previous experiments, both the full range of curved-line stimuli and the first half of that range were used.

\section{Results}

Figure 8, sections a and b, shows the result of the curvature acuity measurements for the full-range and first half-range sets of curvedline stimuli, respectively. The percentage of correct responses, corresponding to the correct discrimination of the more curved line (curvature parameter $s+d s$ ) from the less curved line (curvature parameter $s-d s$ ), is plotted against the reference value of the curvature parameter $s$. Results are pooled over subjects. The magnitude of the increment $d s$ was fixed at $.17 \mathrm{~min}$ arc in all cases.

Although there was a small and systematic decrease in discrimination performance with increasing value of the parameter $s$ for the full range of curved-line stimuli (Figure 8, section a), departures from linearity were small. The broken line fitted to the points is a least squares regression line, gradient $-3.32 \%$ min-arc ${ }^{-1}$, which gives a good fit $\left(\chi^{2}(5)=4.04, p>.5\right)$. The gradient is significantly different from zero $(z=2.60, p<.01)$. For the half-range stimulus set (Figure 8, section b), deviations from linearity were also small. The broken line is a least squares regression line, gradient $2.24 \%$ min-arc ${ }^{-1}$, with excellent fit $\left(\chi^{2}(5)=2.80, p>\right.$ $.5)$. The gradient was not significantly different from zero $(z=1.13, p>.2)$. This linearity in performance was unlikely to be the result of

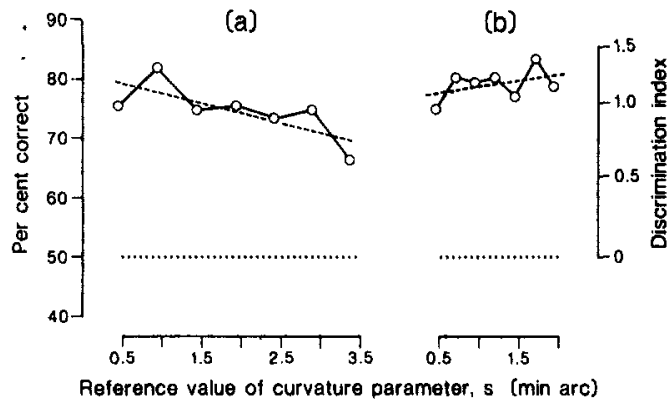

Figure 8. Curvature acuity in an array of two lines. (The percentage of correct responses for discriminating the more curved line, curvature parameter $s+d s$, from the less curved line, curvature parameter $s-d s$, is plotted against reference value of the curvature parameter $s$. The magnitude of the increment $d s$ was fixed at $.17 \mathrm{~min}$ arc. Data were pooled over four subjects. Performance in section a refers to the full range of curved-line stimuli and in section $b$ to the first half of that range. Chance-level performance is shown by the dotted line. The broken line is a least squares regression line.)

ceiling effects: Performance with the full range of curved-line stimuli declined to a $d^{\prime}$ value of .60 , which was less than the maximum $d^{\prime}$ value of .74 obtained in the 4-curved-lines discrimination paradigm of Experiment 1. Although the full- and half-range data had different gradients overall, the gradient for the first half of the full-range data and the gradient for the half-range data were not significantly different $(z=.92, p>.2)$.

\section{Discussion}

These results on curvature acuity are different from the results of Experiment 1, despite the identity in the range of stimuli presented. Variation in performance here, in contrast to that in Experiment 1, was smooth with reference value of the curvature parameter $s$ and was not significantly different from a linear function. Even when overall level differences were taken into account, the differences between the present data and those of Experiment 1 for the full-range stimulus set reached significance $\left(\chi^{2}(6)=14.79, p<.05\right.$; Figure 8 , section a, and Figure 2, section a).

The fact that there was a marked qualitative change in discrimination performance with change in experimental conditions had been anticipated. As already mentioned, Beck and Ambler $(1972,1973)$ have described qualitative as well as quantitative changes in discrim- 
ination of differences in line slope and line arrangement that depend on the number of figures in the field and on mask delay. Under rather different conditions, Westheimer and Hauske (1975) have shown that the introduction of an additional figure near the test figure interferes with hyperacuity performance. Although mediated by central processes (Westheimer \& Hauske, 1975), these operate over distances much shorter than those involved here. In any event, the variations in discrimination performance with $s$ obtained in Experiment 1 appear not to be attributable to nonuniformities in the curved-line continuum, at least with regard to acuity.

\section{General Discussion}

\section{Overview of Experiments 1-4}

A continuum of curved-line stimuli was generated by an algorithm that secured a transformational uniformity of the scale. Discriminability of pairs of curved lines, separated by a constant distance $2 d s$ on this scale, was measured at successive points $s$ along the scale. In Experiment 1, four curved lines were presented in a 100-msec display followed by a poststimulus mask. Discrimination performance for full-range and half-range sets of curved-line stimuli varied nonsmoothly with s. There were two peaks, the one at $.87 \mathrm{~min}$ arc and the other at $1.81 \mathrm{~min}$ arc.

In Experiment 2, subjects performed a categorical identification experiment in which, with the aid of a poststimulus cue, they labeled the curved-line stimuli of Experiment 1 as straight, just curved, and more than just curved. From these data, a theoretical discrimination performance was computed and shown to be closely congruent to the observed discrimination performance of Experiment 1. Predicted and observed discrimination performances also varied in the same way with changes in the range of stimuli sampled. Experiment 3 showed that two-, three-, and fourinterval rating scales each provided a predicted discrimination performance rather less accurate than that provided by the categorical identification scale. These rating data did, however, show that under the conditions of the rating-scale experiment the curved-line continuum was perceptually uniform: Mean rat- ings increased linearly with curvature parameter $s$.

A further test of scale uniformity was made in Experiment 4 by determining acuity for curvature at successive points along the curved-line continuum. Just two curved lines were presented in each display for a duration of $2 \mathrm{sec}$ with no poststimulus mask. Under these conditions, performance varied linearly with curvature parameter $s$. This result and the results of Experiment 3 implied that the peaks in discrimination performance shown in Experiment 1 were not attributable to nonuniformities in the curved-line continuum.

\section{Two Modes of Visual Pattern Processing}

The present experiments have shown that under conditions in which attention is distributed over a number of elements in the field and in which viewing and effective visual processing time are restricted, performance in discriminating curved-line stimuli was characterized by relatively coarse, discrete processes, corresponding to the categories straight, just curved, and more than just curved. This mode of visual processing may be contrasted with that apparently used when the number of stimuli to be attended to was small and viewing time was effectively unlimited. Here performance was characterized by smooth, continuous, fine-grained processes.

In a number of studies, Beck (1972; Beck \& Ambler, 1972, 1973) has pointed out the special role of "focal" and "distributed" attention in peripheral viewing; similar considerations almost certainly apply here, although with $1.4^{\circ}$ eccentricity the present stimuli were within the central fovea. The properties revealed by Beck for peripheral form discrimination under uncertainty show performance dominated by extremely coarse discrete encodings: Orientation rather than configuration is the decisive factor in discrimination of simple arrangements of line stimuli.

The present experiments have shown a more detailed discrete encoding of curved-line stimuli and add to previous data suggesting other discrete processes encoding the collinearity or noncollinearity of three-dot figures (Foster, $1979,1982 \mathrm{~b}$ ), and the acuteness or obtuseness of chevron figures (Foster, 1980b).

A rationale for the use of discrete or con- 
tinuous encoding processes by the visual system can be contrived on the basis of elementary notions from information theory (see Foster, 1982a; Rosch, 1978). Suppose that there is a restricted picture encoding and processing capacity (lower than that corresponding to all possible receptor-distinguishable light distributions); that is, the product of the number of pattern elements in the field and the number of attribute values that may be assigned one at a time to each element in the field is relatively limited. When there are several pattern elements in the field, each element may be assigned one of only a small number of possible attribute values, whereas when there are a small number of elements in the field, each element may be assigned one of a correspondingly large number of possible attribute values. In the former case the encoding is essentially discrete, whereas in the latter it is essentially continuous.

\section{Long-Term Stability of Categorical Discrimination}

The lability of the categorical discrimination obtained in Experiment 1 has already been noted: It occurred in relation to the effects of stimulus range (see Figure 2, sections a and b) and in relation to the number of curved lines in the display and the effective display duration (see Figure 8, sections a and $b$ ). The evidence that categorical discrimination is dependent on factors such as stimulus range and mode of presentation and that it involves probabilistic processes (Foster, 1980b, 1982a; Experiment 2 here) does not, however, imply that the characteristics of the underlying discrete encodings are themselves necessarily unstable.

To test long-term stability, the curved-line discrimination experiment of Experiment 1 was repeated with the same subjects 1 year later. Both the full-range and half-range stimulus sets were used. No significant change in performance was detected (for the full-range stimulus set, $\chi^{2}(6)=3.29, p>.5$; for the halfrange stimulus set, $\chi^{2}(6)=1.17, p>.5$ ). Thus, although the types of discrete encoding for curved lines may apparently be selected according to the immediate constraints on task and conditions of viewing, their characteristics do have the property of stationarity for the given regime.

\section{Relevance to Visual Pattern Recognition}

Some theories of visual pattern recognition have supposed that the capacity to recognize patterns or objects presented under a variety of spatial transformations, corresponding to changes in stimulus position and orientation and changes in the observer's point of regard, is the result of establishing an internal representation of the pattern or object in terms of various "invariant" attributes or features of that stimulus. The scheme of attributes admissible in such descriptions varies with the theory (see, e.g., the review by Reed, 1973). Typically, local features such as edges, bars, and curves, and global features such as symmetry have been introduced, and, as part of structural description schemes, local features have been specified in conjunction with spatial relations such as "left of," "inside," and "in line with." All of these schemes have the technical property of being "discrete" (Foster, $1980 \mathrm{a})$, in that they involve the construction of an internal representation from a relatively small, finite list of potential properties. The important point about such descriptions is that the internal representation thus formed exhibits an invariance or quasi-invariance to some set or group of spatial transformations of the stimulus.

Support for the existence of these featureand relation-encoding schemes has been adduced on the basis of indirect evidence: Hypothesized internal representations, composed of selected discrete components, have been found to correctly predict performance in matching patterns subjected to large global transformations (see, e.g., Foster \& Mason, 1979; Kahn \& Foster, 1981). As has been argued elsewhere (Foster, 1980a, 1980b), the approach taken in the present study is more direct in its analysis of the components making up these putative internal representations. The identity of components is not inferred from the quasi-invariances in recognition obtained under large pattern transformations but from the discontinuities in discrimination performance obtained under small pattern transformations. It is not necessary that the hypothesized component of the internal representation under consideration need be associated with some spatially isolated figure (as was the case for the array of well-separated 
curved lines used in the present experiments); instead, the technique of generating a continuum of patterns and measuring discrimination of pairs of patterns, separated by fixed distances on the stimulus continuum, may, in principle, be applied to arbitrarily complex patterns or objects. This approach has been developed by Eijkman (Note 2) in the perturbation of pictorial displays.

\section{Other Categorization Processes}

The discrete encodings considered here are low level, and, although labile, as already suggested in relation to task and conditions of stimulus presentation, their characteristics remain stable over long periods. They are not susceptible to voluntary and immediate modification nor are they arbitrary (Rabbitt, 1978). The categories straight, just curved, and more than just curved, which were applied by subjects in the labeling task of Experiment 2 and which apparently underlay the discrimination performance of Experiment 1, were natural in the sense of being determined by successive threshold steps along an internal curved-line continuum. These natural categories should, however, be distinguished from the natural categories discussed by Rosch $(1973,1978)$ in relation to various higher level semantic and cognitive stimulus categorizations.

The operation of categorical processes in high-level form-perception tasks, involving sophisticated attributes related to cultural knowledge, is well known. In one important study, Rosch (1973) used subjects drawn from a Stone Age culture and showed that for simple geometric figures-for example, a square and various transformations of it (not continuously parameterized as in the present study) - it was the "good forms" of Gestalt psychology that were the most easily learned. Cermak (1977) used a smoothly parameterized set of stimulus forms (closed curves generated by a Fourier algorithm; see Shepard \& Cermak, 1973) to explore same-different responses. He showed that pairs of stimuli that were interpreted in the same way, for example, "animal head" or "woman in hat," were confused more often than pairs that were interpreted in different ways.

Eijkman (Note 2) used an extension of the present pattern-perturbation technique to ex- plore the effectiveness of different descriptors for pictorial line stimuli. Line pictures were perturbed by omitting or displacing a variety of components: single pixels, line fragments, contours, and whole figures. Deterioration was measured by a rating procedure. When pictures were perturbed by omission of components, it was lines rather than pixels that proved the more important descriptors. (Omission of contours and whole figures had similar effects to the omission of lines.) This result was compatible with the notion of there being broadly tuned line encodings involved in the early stages of pattern processing. When pictures were perturbed by displacement rather than by omission of components, it was contours that proved most important. Eijkman suggested that in a system in which the output of line-encoding processes is synthesized into contours and these in turn into whole figures, the displacement of contours would be particularly disruptive as new relations would be formed between whole figures in the picture. In contrast, if whole figures were displaced, then most spatial relations would retain their meaning.

Despite the difference in modality, the results of the present study are, in the level at which discrete encoding processes apparently mediate perceptual function, more closely analagous to the findings of Liberman and his colleagues (Liberman, Harris, Hoffman, \& Griffith, 1957; Liberman, Harris, Kinney, \& Lane, 1961) on the phoneme-boundary effect. As in auditory perception (e.g., Pisoni \& Lazarus, 1974), the problem of disconfounding the contributions of discrete and continuous attributes to the encoding of visual stimuli is not trivial (Foster, 1980b). In principle, however, it should be possible to manipulate the conditions of stimulus presentation, as was done here, to bias the use of discrete modes of visual processing. Pattern-perturbation techniques might then be used to analyze other low-level pattern-encoding processes.

\section{Reference Notes}

1. Foster, D. H. Effects of number and mask delay in categorical discrimination of curved lines. Manuscript in preparation, 1983.

2. Eijkman, E. G. J. Perturbing line pictures for identification of visual features and their syntax. Manuscript in preparation, 1983. 


\section{References}

Andrews, D. P., Butcher, A. K., \& Buckley, B. R. Acuities for spatial arrangement in line figures: Human and ideal observers compared. Vision Research, 1973, 13, 599620.

Baker, R. J., \& Nelder, J. A. The GLIM system. Oxford, England: Numerical Algorithms Group, 1978.

Barlow, H. B. The Ferrier lecture, 1980. Critical limiting factors in the design of the eye and visual cortex. Proceedings of the Royal Society, London, Series B, 1981, $212,1-34$.

Beck, J. Similarity grouping and peripheral discriminability under uncertainty. American Journal of Psychology, $1972,85,1-19$.

Beck, J. Similarity grouping of curves. Perceptual and Motor Skills, 1973, 36, 1331-1341.

Beck, J., \& Ambler, B. Discriminability of differences in line slope and in line arrangement as a function of mask delay. Perception \& Psychophysics, 1972, 12, 33-38.

Beck, J., \& Ambler, B. The effects of concentrated and distributed attention on peripheral acuity. Perception \& Psychophysics, 1973, 14, 225-230.

Braida, L. D., \& Durlach, N. I. Intensity perception. II. Resolution in one-interval paradigms. Journal of the Acoustical Society of America, 1972, 51, 483-502.

Cermak, G. W. Performance in a delayed comparison discrimination task as a function of stimulus interpretation. Perception \& Psychophysics, 1977, 21, 69-76.

Cross, D. V., Lane, H. L., \& Sheppard, W. C. Identification and discrimination functions for a visual continuum and their relation to the motor theory of speech perception. Journal of Experimental Psychology, 1965, 70 , 63-74.

Cutting, J. E., \& Rosner, B. S. Categories and boundaries in speech and music. Perception \& Psychophysics, 1974, $16,564-570$.

Durlach, N. I., \& Braida, L. D. Intensity perception. I. Preliminary theory of intensity resolution. Journal of the Acoustical Society of America, 1969, 46, 372-383.

Eriksen, C. W., \& Spencer, T. Rate of information processing in visual perception: Some results and methodological considerations. Journal of Experimental Psychology, 1969, 79, 1-16.

Estes, W. K., \& Taylor, H. A. A detection method and probabilistic models for assessing information processing from brief visual displays. Proceedings of the National Academy of Sciences, 1964, 52, 446-454.

Estes, W. K., \& Taylor, H. A. Visual detection in relation to display size and redundancy of critical elements. Perception \& Psychophysics, 1966, 1, 9-16.

Farrell, J. E., \& Shepard, R. N. Shape, orientation, and apparent rotational motion. Journal of Experimental Psychology: Human Perception and Performance, 1981, 7, 477-486.

Foster, D. H. Discrete internal pattern representations and visual detection of small changes in pattern shape. Perception \& Psychophysics, 1979, 26, 459-468.

Foster, D. H. A description of discrete internal representation schemes for visual pattern discrimination. Biological Cybernetics, 1980, 38, 151-157. (a)

Foster, D. H. A spatial perturbation technique for the investigation of discrete internal representations of visual patterns. Biological Cybernetics, 1980, 38, 159-169. (b)

Foster, D. H. Analysis of discrete internal representations of visual pattern stimuli. In J. Beck (Ed.), Organization and representation in perception. Hillsdale, N.J.: Erlbaum, 1982. (a)

Foster, D. H. The dependence of observer characteristics upon image complexity. Electronic image processing (IEE Conference Proceedings No. 214) London: Institution of Electrical Engineers, 1982. (b)

Foster, D. H., \& Mason, R. J. Transformation and relational-structure schemes for visual pattern recognition. Biological Cybernetics, 1979, 32, 85-93.

Fox, J. Continuity, concealment and visual attention. In G. Underwood (Ed.), Strategies of information processing. London: Academic Press, 1978.

Green, D. M., \& Swets, J. A. Signal detection theory and psychophysics. New York: Wiley, 1966.

Guilford, J. P. Psychometric methods. New York: McGrawHill, 1954.

Hakiel, S. R. Variable and constant errors of perceived angle size. Unpublished doctoral dissertation, University of Keele, Staffordshire, England, 1978.

Healy, A. F., \& Repp, B. H. Context independence and phonetic mediation in categorical perception. Journal of Experimental Psychology: Human Perception and Performance, 1982, 8, 68-80.

Julesz, B. Foundations of Cyclopean perception. Chicago: University of Chicago Press, 1971.

Julesz, B. Spatial nonlinearities in the instantaneous perception of textures with identical power spectra. Philosophical Transactions of the Royal Society, London, Series B, 1980, 290, 83-94.

Kahn, J. I., \& Foster, D. H. Visual comparison of rotated and reflected random-dot patterns as a function of their positional symmetry and separation in the field. Quarterly Journal of Experimental Psychology, 1981, 33A, 155-166.

Liberman, A. M., Harris, K. S., Hoffman, H. S., \& Griffith, B. C. The discrimination of speech sounds within and across phoneme boundaries. Journal of Experimental Psychology 1957, 54, 358-368.

Liberman, A. M., Harris, K. S., Kinney, J. A., \& Lane, $H$. The discrimination of relative onset-time of the components of certain speech and nonspeech patterns. Journal of Experimental Psychology, 1961, 61, 379388.

Ludvigh, E. Direction sense of the eye. American Journal of Ophthalmology, 1953, 36, 139-143.

Lupker, S. J., \& Massaro, D. W. Selective perception without confounding contributions of decision and memory. Perception \& Psychophysics, 1979, 25, 60-69.

Macmillan, N. A., Kaplan, H. L., \& Creelman, C. D. The psychophysics of categorical perception. Psychological Review, 1977, 84, 452-471.

Massaro, D. W. Auditory information processing. In W. K. Estes (Ed.), Handbook of learning and cognitive processes: Vol. 4. Attention and memory. Hillsdale, N.J.: Erlbaum, 1976.

Morotomi, T. Selective reduction in visibility of a posttarget by an identical pretarget masked by noise. Perception \& Psychophysics, 1981, 30, 594-598.

Olson, R. K., \& Attneave, F. What variables produce similarity grouping? American Journal of Psychology, 1970, $83,1-21$.

Onley, J. W., \& Volkmann, J. The visual perception of perpendicularity. American Journal of Psychology, 1958, $71,504-516$ 
Parducci, A. Contextual effects: A range-frequency analysis. In E, C. Carterette \& M. P. Friedman (Eds.) Handbook of perception: Vol. 2. Psychophysical judgment and measurement. New York: Academic Press, 1974.

Pisoni, D. B., \& Lazarus, J. H. Categorical and noncategorical modes of speech perception along the voicing continuum. Journal of the Acoustical Society of America, $1974,55,328-333$.

Pomerantz, J. R. Are complex visual features derived from simple ones? In E. L. J. Leeuwenberg \& H. F. J. M. Buffart (Eds.), Formal theories of visual perception, Chichester, England: Wiley, 1978.

Poulton, E. C. Models for biases in judging sensory magnitude. Psychological Bulletin, 1979, 86, 777-803.

Rabbitt, P. Sorting, categorization, and visual search. In E. C. Carterette \& M. P. Friedman (Eds.), Handbook of perception: Vol. 9. Perceptual processing. New York: Academic Press, 1978.

Reed, S. K. Psychological processes in pattern recognition. New York: Academic Press, 1973.

Repp, B. H., Healy, A. F., \& Crowder, R. G. Categories and context in the perception of isolated steady-state vowels. Journal of Experimental Psychology: Human Perception and Performance, 1979, 5, 129-145.

Rochlin, A. M. The effect of tilt on the visual perception of parallelness. American Journal of Psychology, 1955, 68, 223-236.

Rosch, E. H. Natural categories. Cognitive Psychology, 1973, 4, 328-350.

Rosch, E. Principles of categorization. In E. Rosch \& B. B. Lloyd (Eds.), Cognition and categorization. Hillsdale, N.J.: Erlbaum, 1978.
Shepard, R. N., \& Cermak, G. W. Perceptual-cognitive explorations of a toroidal set of free-form stimuli. Cognitive Psychology, 1973, 4, 351-377.

Stevens, S. S. Perceptual magnitude and its measurement. In E. C. Carterette \& M. P. Friedman (Eds.). Handbook of perception. Vol. 2. Psychophysical judgment and measurement. New York: Academic Press, 1974.

Studdert-Kennedy, M., Liberman, A. M., Harris, K. S., \& Cooper, F. S. Motor theory of speech perception: A reply to Lane's critical review. Psychological Review, 1970, 77, 234-249.

Swets, J. A. The relative operating characteristic in psychology. Science, 1973, 182, 990-1000.

Watt, R. J., \& Andrews, D. P. Contour curvature analysis: Hyperacuities in the discrimination of detailed shape. Vision Research, 1982, 22, 449-460.

Weene, P., \& Held, R. Changes in perceived size of angle as a function of orientation in the frontal plane. Journal of Experimental Psychology, 1966, 71, 55-59.

Westheimer, G. The spatial sense of the eye. Investigative Ophthalmology and Visual Science, 1979, 18, 893-912.

Westheimer, G. Visual hyperacuity. Progress in Sensory Physiology, 1981, 1, 1-30.

Westheimer, G., \& Hauske, G. Temporal and spatial interference with vernier acuity. Vision Research, 1975, $15 ; 1137-1141$

Westheimer, G., \& McKee, S. P. Spatial configurations for visual hyperacuity. Vision Research, 1977, 17, 941-947.

Wood, C. C. Discriminability, response bias, and phoneme categories in discrimination of voice onset time. Journal of the Acoustical Society of America, 1976, 60, 13811389.

\section{Appendix}

\section{Derivation of Predicted Discrimination Performance From Categorical Identification or Categorical Rating Performance}

For each curved line in the family of curved lines illustrated in Figure 1 (section a) define a Cartesian coordinate system with origin fixed at the lowest point of the curve. Let $c$ be the most curved line, curvature parameter $s_{1}=3.79 \mathrm{~min}$ arc, as shown in Figure 1 (section a). Let $\left(x_{1}, y_{1}\right), x_{1}=x_{1}\left(y_{1}\right)$, represent $c$. Any member $\left(x^{\prime}, y^{\prime}\right)$ of the family of curved lines (Figure 1, section a) could be obtained from any other member $(x, y)$ by the action of some transformation $T_{s}$ drawn from a local 1-parameter group of local transformations, thus:

$$
\left(x^{\prime}, y^{\prime}\right)=T_{s}(x, y)=\left(\left(s / s_{1}\right) x_{1}(y)+x, y\right),
$$

where $s$ runs over the given range. The group property of the transformations $T_{s}$ means that $T_{s+l}(x, y)=T_{s}\left(T_{t}(x, y)\right)$, which gives the $s$ scale the property of transformational uniformity referred to in the text. In particular, a fixed increment $d s$ in $s$ results in a transformation $T_{d s}$, the action of which is independent of $s$; that is,

$$
T_{s+d s}(x, y)=T_{d s}\left(T_{s}(x, y)\right) .
$$

For further details and more precise definitions, see Foster (1980b).

For each $s$ in the given range, let $p_{i}\left(T_{s}(x, y)\right)=$ $p_{i}(s)$ be the probability that categorical label $r_{i}$, $1 \leqslant i \leqslant n$, is assigned to the curved line $T_{s}(x, y)$. For Experiment 2, $n=3$; for Experiment 3, $n=$ $2,3,4$. The visual discriminability of two curved lines $T_{s+d s}(x, y)$ and $T_{s-d s}(x, y)$ differing in curvature parameter by $2 d s$ is assumed (Foster, 1980b) to be determined by a monotonic increasing function of the differences between the assignment probabilities of the internal representations produced by the two curved lines. Suppose, critically, that these assignment probabilities are the same as those obtained in the given categorial labeling experiment. The difference $e(s)$ between the two sets of probabilities $p_{i}(s+d s)(1 \leqslant i \leqslant n)$ and $p_{i}(s-d s)(1 \leqslant i \leqslant n)$ may be quantified naturally with the $l_{p}^{n}$ norm:

$$
e(s)=\left(\sum_{i=1}^{n}\left|p_{i}(s+d s)-p_{i}(s-d s)\right|^{q}\right)^{1 / q},
$$

where $q$ is fixed and $1 \leqslant q<\infty$. When $q=1$, this 


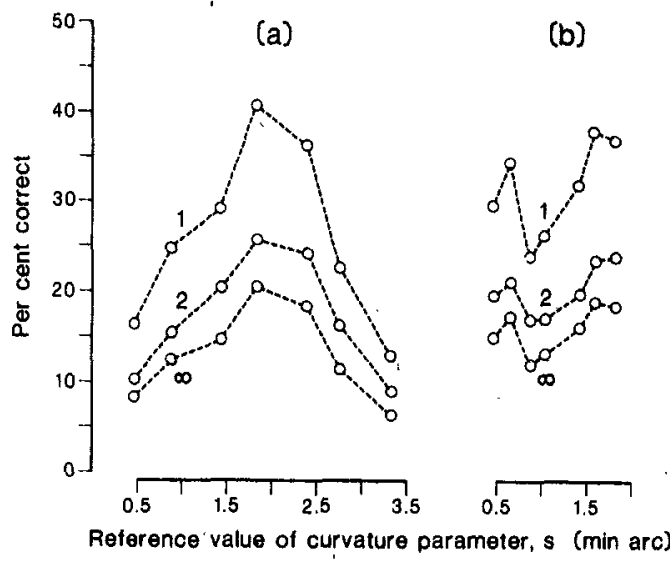

Figure A1. Effect of index $q$ (see the Appendix) on predicted performance in discriminating curved lines. (The percentage of correct responses for discriminating the odd curved line, curvature parameter $s+d s$ [or $s-d s$ ], from the three identical curved lines, curvature parameter $s-$ $d s$ [or, respectively, $s+d s$ ], is plotted against the reference value of the curvature parameter $s$. Performance in section a refers to the full range of curved-line stimuli, and in section $b$ to the first half of that range. Data for predicted discrimination performances were derived from the corresponding sets of categorical identification data of Figure 3 by means of the procedure described in the Appendix. The value of the index $q$ used in the computation of predicted discrimination performance is indicated.) is the city-block metric, and, when $q=2$, the Euclidean metric. Suppose $q=1$. Predicted discrimination performance is then simply the sum over all categories of the absolute differences between assignment proportions for each successive pair of curved lines. In principle, if the categories are sufficiently disjoint, the maximum and minimum values of $e(s)$ are 2.0 and 0 , respectively, the former arising from the absolute difference in proportions being counted effectively twice at category boundaries. For this reason, computed values were divided by 2.0 . The predicted discrimination performances computed from the categorical identification data (Experiment 2) and from the three sets of categorical rating-scale data (Experiment 3) were derived with $q=1$. In Foster (1980b) it is shown that under certain conditions the qualitative characteristics of the dependence of predicted discrimination performance $e(s)$ on $s$ do not depend on the particular choice of $q$. For the present case, the similarity in the dependence of $e(s)$ on $s$ for $q=1,2$, and $\infty$ (the sup norm) is shown in Figure Al.

An informal account of some of the principles underlying the preceding derivation is given in Foster (1982a). A more formal treatment is given in Foster (1980a, 1980b).

Received February 11, 1983

Revision received May 9, 1983

\section{Third Edition of the Publication Manual}

APA has just published the third edition of the Publication Manual. This new edition replaces the 1974 second edition of the Manual. The new Manual updates APA policies and procedures and incorporates changes in editorial style and practice since 1974. It amplifies and refines some parts of the second edition, reorganizes other parts, and presents new material. (See the March issue of the American Psychologist for more on the third edition.)

All manuscripts to be published in the 1984 volumes of APA's journals will be copy edited according to the third edition of the Manual. Therefore, manuscripts being prepared now should be prepared according to the third edition. Beginning in 1984, submitted manuscripts that depart significantly from third edition style will be returned to authors for correction.

The third edition of the Publication Manual is available for $\$ 12$ for members of APA and $\$ 15$ for nonmembers. Orders of $\$ 25$ or less must be prepaid. A charge of $\$ 1.50$ per order is required for shipping and handling. To order the third edition, write to the Order Department, APA, 1400 N. Uhle Street, Arlington, VA 22201. 